\title{
Uso do nióbio como refinador de grão para ligas de aluminio-silício
}

\section{Use of niobium as grain refiner for aluminum silicon alloys}

\author{
1 Alexandre Leão Quadro aleao@amg-al.com \\ 1 Marco Túlio Raposo \\ 2 Antônio Luiz Ribeiro Sabariz
}

1 Programa de Pós-Graduação em Física e Química de Materiais - FQMat, Universidade Federal de São João Del Rei , UFSJ.
2 Departamento de Engenharia Mecânica - DEMEC, Universidade Federal de São João Del Rei, UFSJ.

\section{Resumo}

Com o objetivo de encontrar alternativas para o refino de grão de ligas alumínio-silício, um novo refinador foi experimentalmente produzido à base de nióbio, boro e alumínio (Al-3\%Nb-1\%B). Essa nova liga foi caracterizada quimicamente por espectroscopia de emissão óptica por plasma acoplado indutivamente (ICP-OES), microestruturalmente por microscopia eletrônica de varredura (MEV) e por espectrometria de energia dispersiva (EDS). Foi utilizado o teste TP1, da Aluminum Association, para medir a capacidade de refino dessa nova liga sobre a liga Al- $11 \% \mathrm{Si}-$ $0,1 \% \mathrm{Mg}$ e compará-la com o refinador convencional TiBAl 5/1 (5\% de titânio e 1\% de boro). 0 tamanho de grão foi medido com uma lupa estereoscópica, utilizando-se o método do intercepto da ABNT. Para os cinco minutos iniciais, o teste TP1 realizado com $1 \mathrm{~kg} / \mathrm{t}$ da liga Al-3\% $\mathrm{Nb}-1 \% \mathrm{~B}$ apresentou tamanhos de grãos semelhantes ao teste realizado com $5 \mathrm{~kg} / \mathrm{t}$ de TiBAl $5 / 1$, indicando que esse novo refinador pode ser utilizado industrialmente para o refino de grão de ligas alumínio-silício, desde que o tempo de residência seja próximo de 5 minutos.

\section{Palavras-chave}

Refinador; nióbio; alumínio.

\begin{abstract}
In order to find alternatives for grain refining of aluminum-silicon alloys, a new grain refiner based on niobium, boron and aluminum (Al-3\%Nb-1\%B) was experimentally produced. This new alloy was chemically characterized by Inductively Coupled Plasma Optical Emission Spectrometry (ICP-OES) and microstructurally, by means of scanning electron microscopy (SEM) and energy dispersive spectrometry (EDS). The TP1 test from Aluminum Association was used to measure the refining capacity of this new alloy on the alloy $\mathrm{Al}-11 \% \mathrm{Si}-0.1 \% \mathrm{Mg}$ and compare it to the conventional refiner TibAl 5/1 (5\% of titanium and $1 \%$ of boron). The grain size was measured with a stereoscopic microscope using the intercept method from ABNT (Brazilian standards). For the first five minutes, the TP1 test performed with $1 \mathrm{~kg} / \mathrm{t}$ of Al-3\% $\mathrm{Nb}-1 \% \mathrm{~B}$ showed grain sizes similar to the test with $5 \mathrm{~kg} / \mathrm{t}$ of TibAl 5/1, indicating that this new grain refiner can be industrially used for grain refining of aluminum-silicon alloys, since the residence time is around 5 minutes.
\end{abstract}

\section{Keywords}

Refiner; niobium; aluminum.

\section{Como você deve citar?}

QUADRO, Alexandre Leão; RAPOSO, Marco Túlio; SABARIZ, Antônio Luiz Ribeiro. Uso do nióbio como refinador de grão para ligas de aluminio-silício. Cadernos UniFOA, Volta Redonda, n. 30, p. 37-45, abr. 2016. 


\section{INTRODUÇÃO}

O refino de grão é uma prática comum e antiga na indústria do alumínio, pois melhora as propriedades mecânicas, o acabamento superficial e reduz as tensões residuais internas (GREER et al., 2000; QUESTED, 2004; ZHANG et al., 2005; ARANGO et al., 2012). 0 método mais utilizado mundialmente é o refino do grão através da adição de ligas refinadoras à base de alumínio, titânio e boro (TiBAI) (EASTON et al., 1999; QIU et al., 2007; ARANGO et al., 2012). Essas ligas contém dois tipos de partículas. 0 primeiro é o diboreto de titânio $\left(\mathrm{TiB}_{2}\right)$, que é um material cerâmico muito estável e inerte, com tamanho entre $1 \mathrm{a}$ 2 microns, com uma estrutura hexagonal que não se dissolve no alumínio. 0 segundo é o alumineto de titânio $\left(\mathrm{TiAl}_{3}\right)$, com tamanhos na faixa de 30 a 50 microns e que se dissolve rapidamente no alumínio fundido. Desse modo, um grande número de partículas de $\mathrm{TiB}_{2}$ está disperso no metal fundido para funcionar como centros ativos de nucleação durante a solidificação. Esse mecanismo é conhecido como nucleação heterogênea (ARANGO et al., 2012). 0 tamanho de grão inicialmente diminuiu em função do tempo de contato, o que é atribuído à dispersão das partículas de $\mathrm{TiB}_{2}$ e pela dissolução das partículas de $\mathrm{TiAl}_{3}$, entretanto um aumento no tamanho de grão (conhecido como fade) é observado em tempos de espera mais longos (QUESTED, 2004).

É bem conhecido que certos elementos químicos podem afetar negativamente a eficiência dessas ligas refinadoras, o que é chamado de envenenamento do refino de grão. 0 silício é um desses elementos e, para ligas de fundição baseadas em alumínio-silício, nota-se que a partir de $2 \%$ a $3 \%$ de silício inicia-se o efeito de envenenamento, que se agrava com o aumento desse teor (QIU et al., 2007; ARANGO et al., 2012; BIROL, 2012). Esse efeito de envenenamento ocasionado pelo silício é provavelmente devido à formação de uma camada de $\mathrm{Ti}_{5} \mathrm{Si}_{3}$ sobre as partículas de $\mathrm{TiAl}_{3}$, inibindo as suas propriedades refinadoras (QIU et al., 2007).

Desde que as ligas Al-Si possuem boas propriedades de fundição, soldabilidade e resistência à corrosão, elas compõem a base da mais popular família de ligas de fundição na indústria do alumínio (QIU et al., 2007; CHEN et al., 2012; BIROL, 2014). Várias tentativas foram feitas para melhorar a resposta ao refino de grão dessas ligas Al-Si nas últimas décadas, principalmente porque um tamanho de grão mais fino melhora o preenchimento do molde, resultando em menos porosidades e quebras à quente (QUESTED et al, 2004; QIU et al., 2007; Nowak et al., 2015). Uma maneira utilizada para melhorar esse refino de grão nas ligas Al-Si é aumentar a quantidade da adição de ligas refinadoras TiBAl (QIU et al., 2007; CHEN et al., 2012), porém não é uma boa solução sob o ponto de vista econômico (QIU et al., 2007; BIROL, 2014).

Segundo Mohant e Gruszleski (1994), empilhamentos atômicos se tornam favoráveis quando os átomos são de tamanho similar. Tal similaridade promove um empacotamento com a mínima distorção na rede e proporciona ligações mais fortes entre os vizinhos. A Figura 1 apresenta um índice qualitativo da performance de vários elementos de transição como nucleantes, quando adicionados como boretos (MOHANTY et al., 1994). Ainda, segundo os mesmos autores, boretos de nióbio, titânio e tântalo apresentam a maior performance qualitativa de nucleação da fase primária Al-alfa.

Para este trabalho foi produzida experimentalmente uma nova liga refinadora à base de nióbio, boro e alumínio (Al-3\% Nb-1\%B), com o objetivo de verificar o seu poder de refino sobre a fase primária Al-alfa da liga Al- $11 \% \mathrm{Si}-0,1 \% \mathrm{Mg}$ (comumente utilizada para a produção de rodas automotivas) e compará-lo com o refino proporcionado pela liga convencional TiBAI 5/1. 
Figura 1 - Performance qualitativa da habilidade de refino dos metais de transição no alumínio.

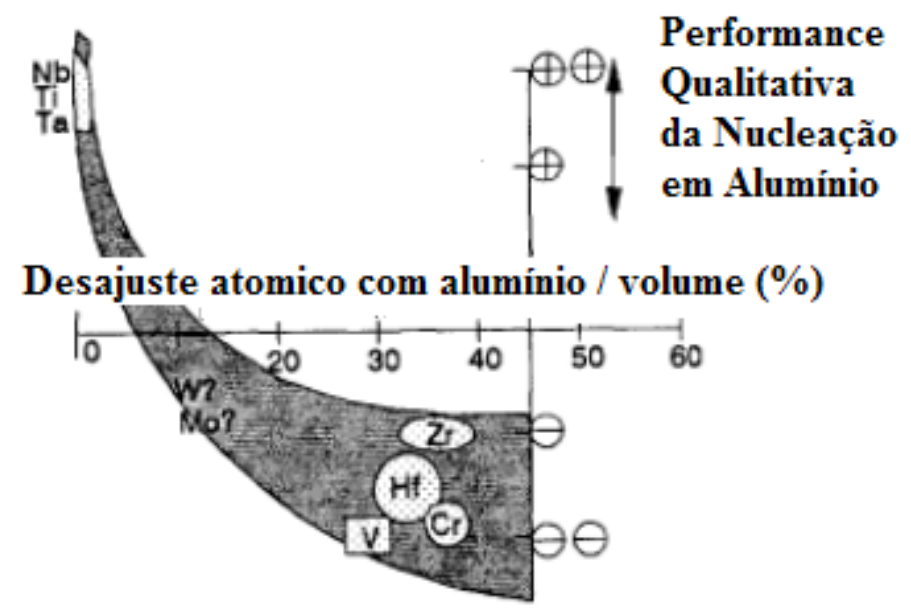

Fonte: (MOHANTY et al., 1994)

\section{MATERIAIS E MÉTODOS}

\subsection{Produção da Liga Refinadora Al-3\%Nb-1\%B}

2.1.1 Materiais

Para a produção experimental da liga refinadora $\mathrm{Al}-3 \% \mathrm{Nb}-1 \% \mathrm{~B}$, foram utilizadas as seguintes matérias-primas:

- 2.500 g de Alumínio primário comercial grau P1020;

- $113 \mathrm{~g}$ de $\mathrm{Nb}_{2} \mathrm{O}_{5}-99,9 \%$ de pureza (como fonte de nióbio);

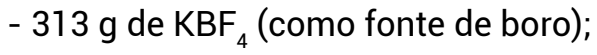

- $782 \mathrm{~g}_{\text {de }} \mathrm{KAIF}_{4}$ (com a finalidade de retirar o $\mathrm{Al}_{2} \mathrm{O}_{3}$ formado da reação do óxido de nióbio com o alumínio, limpando a liga).

\subsubsection{Equipamentos}

Foi utilizado um forno de laboratório, à resistência elétrica, de potência ativa de $15 \mathrm{~kW}$, com um cadinho de ferro fundido instalado. Para manter a agitação do banho, foi utilizada uma lança de Sialon, com injeção de argônio, conforme desenho esquemático da Figura 2. 
Figura 2 - Esquema da produção experimental da liga Al-3\%Nb-1\%B.

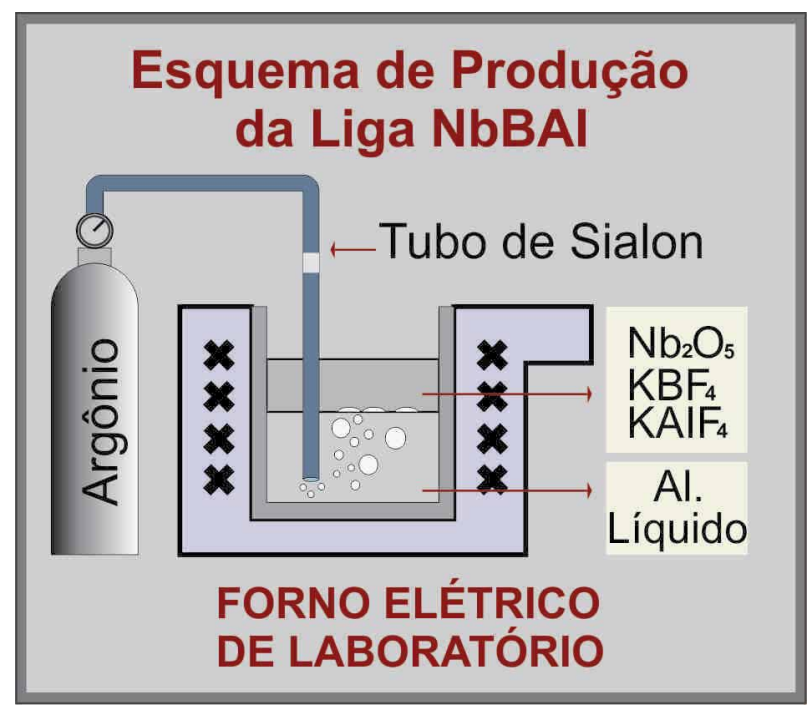

Fonte: Autoria própria.

\subsubsection{Parâmetros do processo}

O alumínio liquido $(2.500 \mathrm{~g})$ foi adicionado ao forno e a temperatura controlada em $850^{\circ} \mathrm{C}+/-10^{\circ} \mathrm{C}$.

As matérias-primas, $\mathrm{Nb}_{2} \mathrm{O}_{5}, \mathrm{KBF}_{4}$ e $\mathrm{KAlF}_{4}$, foram previamente misturadas e adicionadas sobre $\mathrm{O}$ alumínio fundido (Figura 2).

Com injeção de argônio, deixou-se as matérias-primas reagirem por 25 minutos e a temperatura final do banho foi de $900^{\circ} \mathrm{C}$.

$\mathrm{O} \mathrm{KAIF}_{4}$ foi vazado do forno e a liga resultante, $\mathrm{Al}-3 \% \mathrm{Nb}-1 \% \mathrm{~B}$, foi lingotada em um lingoteira de ferro fundido.

\subsubsection{Caracterização da liga Al-3\%Nb-1\%B}

A análise química da liga $\mathrm{Al}-3 \% \mathrm{Nb}-1 \% \mathrm{~B}$ foi feita em um Espectrômetro Indutivamente Acoplado por Plasma modelo iCAP 6000 Series ICP-OES, de propriedade da empresa LSM Brasil SA / AMG Advanced Metallurgical Group.

As fases presentes foram investigadas por um microscópio MEV/EDS, pertencente à Universidade Federal de São João Del Rei, de fabricação da Hitachi e modelo TM 3000.

\subsection{Teste de Refino do Al-3\%Nb-1\%B sobre a Liga Al-11\%Si-0,1\%Mg}

O teste de refino TP1 (ALUMINUM ASSOCIATON, 2012) é um teste que verifica a capacidade de um refinador de grão. Consiste na fusão de $10 \mathrm{~kg}$ da liga a ser refinada, em um forno de laboratório e da adição do refinador de grão, a uma taxa de adição estipulada e temperatura do banho controlada. Amostras são retiradas em tempos pré-determinados, com um molde de aço em formato de cone e resfriadas em um tanque com uma vazão de água de 3,8 litros por minuto (Figura 3). 
Figura 3 - Molde e tanque de resfriamento utilizados para a coleta das amostras.

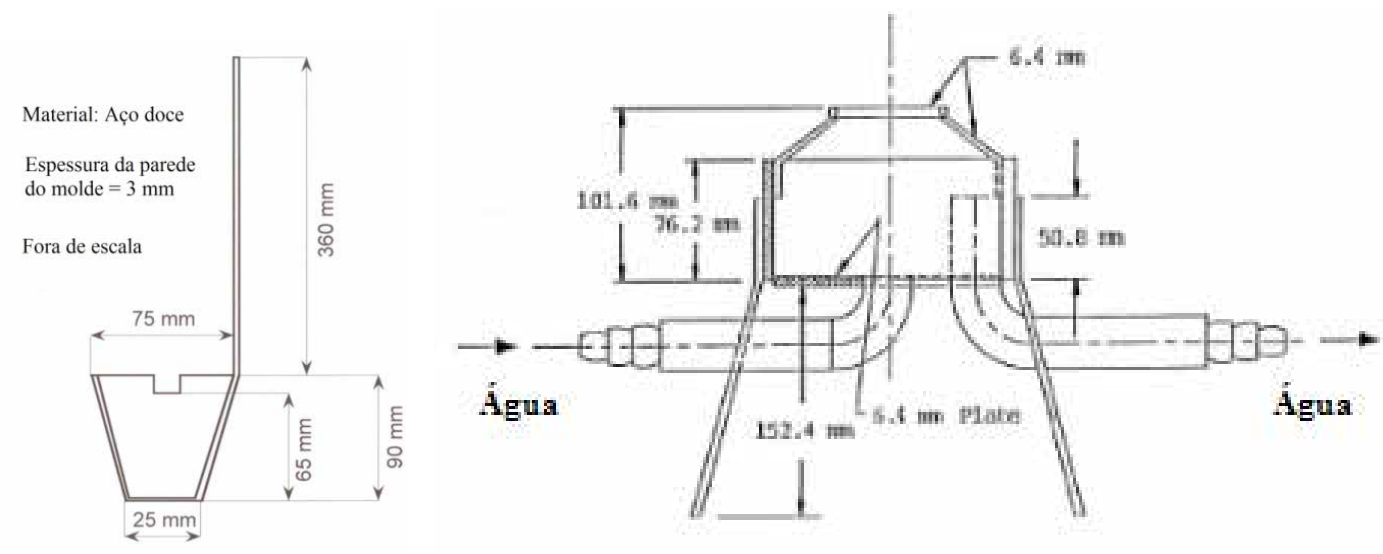

Fonte: Aluminum Association - Teste TP1 (2012).

As amostras resultantes são cortadas a 38 milímetros da base menor do cone, e atacadas por 20 segundos, com solução ácida de Poulton $\left(60 \% \mathrm{HCl}\right.$ a $37 \% ; 30 \% \mathrm{HNO}_{3}$ a $65 \% ; 5 \% \mathrm{HF}$ a $50 \%$ e $5 \%$ de água) e clareadas com a solução: $67 \%$ de $\mathrm{HNO}_{3}$ a $65 \% ; 20 \%$ de $\mathrm{HF}$ a $50 \%$ e $13 \%$ de água, para revelar os grãos, que são medidos no plano de corte, através de uma lupa estereoscópica e utilizando-se o método do intercepto da ABNT (ABNT NBR 11568, 1990).

Realizou-se, em triplicata, testes de refino TP1, utilizando-se uma taxa de adição de $1 \mathrm{~kg} / \mathrm{t}$ para o novo refinador $\mathrm{Al}-3 \% \mathrm{Nb}-1 \% \mathrm{~B}$ na liga $\mathrm{Al}-11 \% \mathrm{Si}-0,1 \% \mathrm{Mg}$. Foram retiradas amostras nos tempos: zero (sem adição), 5, 15, 30, 45, 60, 90, 120, 150, 180 e 240 minutos após a adição do refinador. Para efeitos comparativos, os mesmos testes foram feitos, utilizando-se a taxa de adição de $5 \mathrm{~kg} / \mathrm{t}$ do refinador convencional TiBAI 5/1 (5\% de Titânio e 1\% Boro). A temperatura utilizada para a realização dos testes foi de $680+/-10^{\circ} \mathrm{C}$.

\subsubsection{Liga Al-11\%Si-0,1\%Mg}

Para cada teste, foi utilizada a liga Al- $11 \% \mathrm{Si}-0,1 \% \mathrm{Mg}$, com a composição química objetivada, conforme Tabela 1.

Utilizou-se as seguintes matérias-primas para a produção da liga Al-11\%Si-0,1\%Mg: $8.885 \mathrm{~g}$ de alumínio grau P1020; $1.100 \mathrm{~g}$ de silício metálico grau metalúrgico; $11 \mathrm{~g}$ de magnésio metálico; 5,5 g de TiAl $10 \%$.

Tabela 1 - Composição química objetivada da liga Al-11\%Si-0,1\%Mg (\% em peso)

\begin{tabular}{ccccccc}
\hline$\% \mathrm{Si}$ & $\% \mathrm{Mg}$ & $\% \mathrm{Fe}$ & $\% \mathrm{Sr}$ & $\% \mathrm{Ti}$ & $\% \mathrm{Al}$ & Outros \\
\hline $11,0+/-0,2$ & $0,10+/-0,02$ & $0,10+/-0,03$ & $<0,0010$ & $0,0100+/-0,002$ & Balanço & $0,03 \% \max$ \\
\hline
\end{tabular}

Fonte: Autoria própria. 


\subsection{Análise Química da Liga Al-3\%Nb-1\%B}

A análise química da liga Al-3\%Nb-1\%B, via ICP-OES, apresentou o teor final de nióbio de 2,6\% e o do boro de $0,8 \%$, ficando ambos dentro do limite aceitável para a realização dos testes de refino. 0 teor de ferro foi de $0,6 \%$, provavelmente em virtude da contaminação do cadinho de ferro fundido e da lingoteira, porém não afetará o resultado final dos testes, devido às baixas taxas de adição utilizadas desse refinador.

\subsection{Análise Microestrutural via MEV/EDS da Liga Al-3\%Nb-1\%B}

$\mathrm{Na}$ análise via EDS, foram encontradas, predominantemente, partículas da fase $\mathrm{NbB}_{2}$, com tamanho médio de $1,1 \mathrm{~mm}$, com um tamanho máximo de $2,25 \mathrm{~mm}$, sendo $98 \%$ das partículas menores que $2 \mathrm{~mm}$. Poucas fases de $\mathrm{AlB}_{2}$ foram encontradas. Uma foto da microestrutura pode ser vista na Figura 4, juntamente com o espectro EDS de uma partícula de $\mathrm{NbB}_{2}$. Nowak et al. (2015) produziram ligas

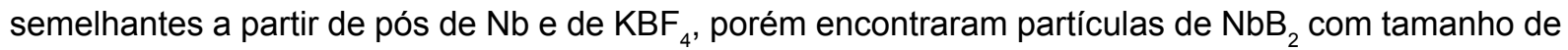
$5 \mathrm{~mm}$ em média, provavelmente devido ao maior tempo de reação utilizado (2 horas).

Figura 4 - Microestrutura da liga Al-3\%Nb-1\%B - 5.000X e Espectro EDS da partícula

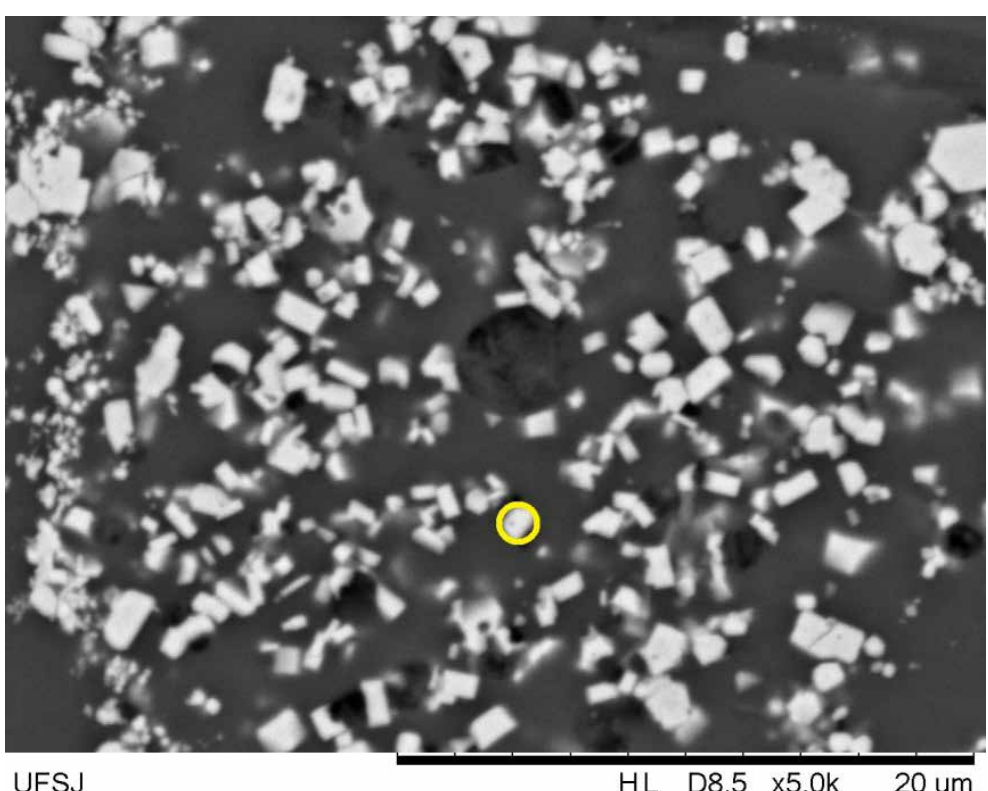

Fonte: Autoria própria (2014)

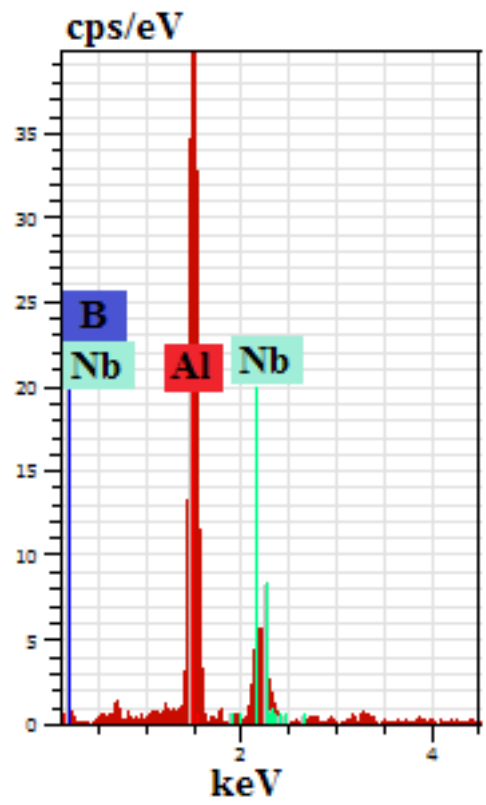

\subsection{Teste de Refino TP 1}

A Tabela 2 apresenta os tamanhos de grãos obtidos nos testes TP1.

Quando não são utilizados os refinadores de grão (tempo zero), a liga Al-11\%Si-0,1\%Mg apresenta grãos grosseiros, com tamanho médio de, aproximadamente, 1 milímetro. Utilizando os refinadores, estes atuam reduzindo significativamente o tamanho de grão, para, aproximadamente, 0,6 milímetros, após 5 minutos da adição, conforme pode ser visto na Figura 5. Nowak et al. (2015) relataram que uma liga contendo $10 \%$ de silício apresentou tamanhos de grão médios de $0,5 \mathrm{~mm}$, com uma adição 
de $0,1 \%$ de $\mathrm{Nb}$ e $0,1 \%$ de $\mathrm{B}$, através de pó de $\mathrm{Nb}$ e de $\mathrm{KBF}_{4}$, valores consistentes com os encontrados neste trabalho. Porém para as amostras retiradas após 15 minutos, o refinador $\mathrm{Al}-3 \% \mathrm{Nb}-1 \% \mathrm{~B}$ perdeu parte de sua capacidade refinadora, apresentando um rápido fade, quando comparado com o refinador convencional TiBAl 5/1, como pode ser visto na Tabela 02.

Tabela 2 - Tamanhos de grão obtidos nos testes TP1 - 1kg/t do refinador Al-3\%Nb-1\%B e $5 \mathrm{~kg} / \mathrm{t} \mathrm{do}$ refinador TiBAl 5/1

\begin{tabular}{|c|c|c|c|c|c|c|c|c|c|c|c|}
\hline & $\begin{array}{l}\text { Zero } \\
\text { min }\end{array}$ & $05 \mathrm{~min}$ & $15 \mathrm{~min}$ & $30 \mathrm{~min}$ & $45 \mathrm{~min}$ & $60 \mathrm{~min}$ & $90 \mathrm{~min}$ & $\begin{array}{l}120 \\
\mathrm{~min}\end{array}$ & $150 \mathrm{~min}$ & $\begin{array}{l}180 \\
\text { min }\end{array}$ & $\begin{array}{l}240 \\
\text { min }\end{array}$ \\
\hline Al3Nb1B (mm) & 1,1 & 0,6 & 0,9 & 0,9 & 0,9 & 1,0 & 1,0 & 0,9 & 1,0 & 1,0 & 1,0 \\
\hline Desvio padrão & 0,1 & 0,1 & 0,1 & 0,01 & 0,02 & 0,1 & 0,1 & 0,1 & 0,02 & 0,2 & 0,04 \\
\hline TiBAl 5/1(mm) & 1,0 & 0,6 & 0,7 & 0,7 & 0,7 & 0,7 & 0,8 & 0,8 & 0,8 & 0,8 & 0,8 \\
\hline Desvio padrão & 0,05 & 0,1 & 0,03 & 0,1 & 0,1 & 0,1 & 0,05 & 0,1 & 0,03 & 0,05 & 0,05 \\
\hline
\end{tabular}

Fonte: Autoria propria (2015)

Figura 5 - Fotos dos grãos sob luz polarizada. a) 1 kg/t Al-3\%Nb-1\%B b) $5 \mathrm{~kg} / \mathrm{t} \mathrm{TiBAl} \mathrm{5/1.}$
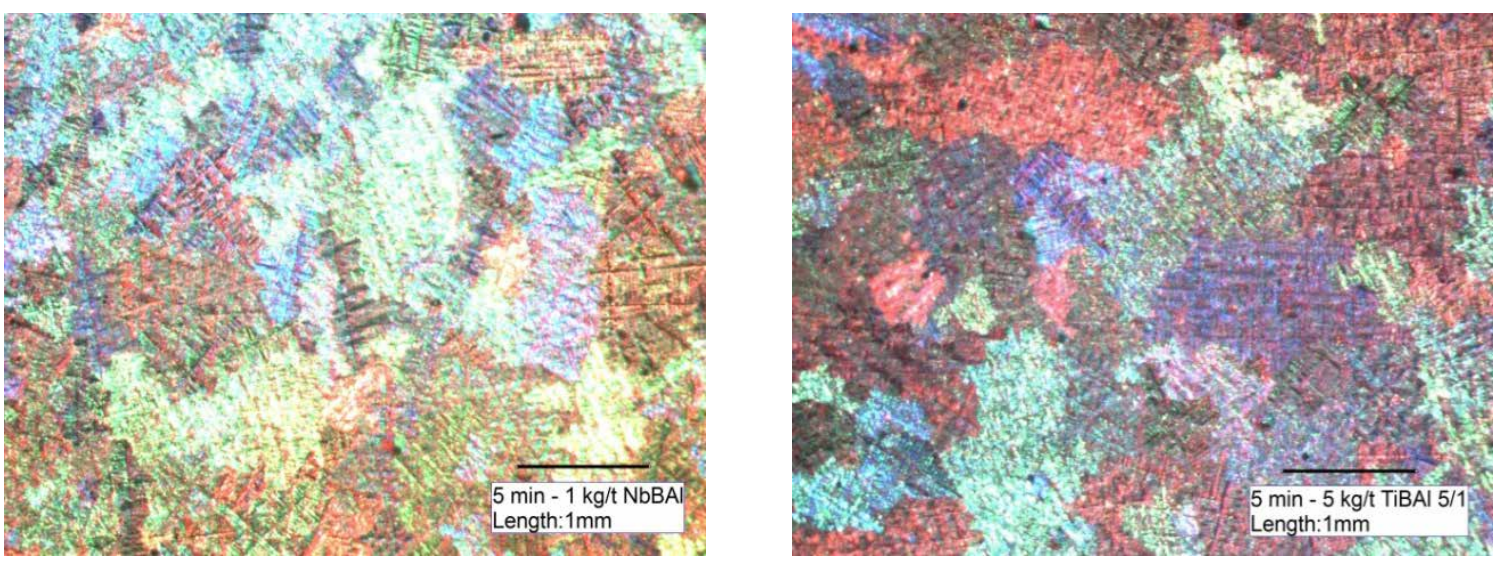

Fonte: Autoria própria (2015)

Segundo Quested (2004), a maioria do fade pode ser atribuído à decantação das partículas nucleantes, entretanto uma agitação do banho recuperaria o poder de refino ao seu nível ótimo. Contudo, no teste TP1 realizado, as amostras são retiradas após a agitação do banho e o motivo do fade da liga $\mathrm{Al}-3 \% \mathrm{Nb}-1 \% \mathrm{~B}$ não pode ser atribuído à decantação das partículas de $\mathrm{NbB}_{2}$.

Ainda segundo Quested (2004), o fade não renovável pode ser atribuído à formação de lama no fundo do forno ou pela aglomeração das partículas inoculadas. Porém, com os dados disponíveis, não foi possível identificar a real causa do fade ocasionado por essa liga.

A nova liga proposta, à base de nióbio, boro e alumínio, pode ser utilizada industrialmente, desde que o tempo entre a adição no banho e a solidificação seja próximo de 5 minutos. Normalmente, esse tempo de residência é obtido na produção industrial, quando o refinador é adicionado nas calhas de vazamento, através da adição controlada, utilizando-se um dosador. 


\section{CONCLUSÕES}

O método proposto para produção experimental da liga refinadora $\mathrm{Al}-3 \% \mathrm{Nb}-1 \% \mathrm{~B}$, utilizando-se $\mathrm{Nb}_{2} \mathrm{O}_{5}, \mathrm{KBF}_{4}, \mathrm{KAlF}_{4}$ e alumínio como matérias-primas, mostrou-se viável.

A fase predominante encontrada na liga $\mathrm{Al}-3 \% \mathrm{Nb}-1 \% \mathrm{~B}$ foi o $\mathrm{NbB}_{2}$, com tamanho médio de 1,1 $\mathrm{mm}$ e tamanho máximo de $2,25 \mathrm{~mm}$.

O tamanho médio dos grãos das amostras da liga Al- $11 \% \mathrm{Si}-0,1 \% \mathrm{Mg}$ retiradas no tempo de 5 minutos no teste TP1 foi de 0,6 milímetros, tanto para $1 \mathrm{~kg} / \mathrm{t}$ do novo refinador conproposto de Al$3 \% \mathrm{Nb}-1 \% \mathrm{~B}$, quanto para $5 \mathrm{~kg} / \mathrm{t}$ do refinador convencional TiBAl $5 / 1$. Porém, a nova liga $\mathrm{Al}-3 \% \mathrm{Nb}-1 \% \mathrm{~B}$ apresentou uma perda na sua capacidade de refino (fade), após 15 minutos da adição.

Devido a esse rápido fade, a nova liga poderá ser utilizada industrialmente, se o tempo entre a adição e a solidificação for próximo de 5 minutos, o que é obtido quando o refinador é adicionado nas calhas de vazamento, através de um dosador.

\section{AGRADECIMENTOS}

Os autores agradecem à LSM Brasil SA / AMG Advanced Metallurgical Group, por disponibilizar os equipamentos e matérias-primas, e à Universidade Federal de São João Del Rei, por disponibilizar o MEV/EDS para caracterização da liga. 


\section{REFERÊNCIAS}

ALUMINUM ASSOCIATION, Standard Test Procedure for Aluminum Alloy Grain Refiners - TP1, 2012.

ARANGO, J. M. R.; MARTORANO, M. A. Efeitos da adição do inoculante Al-3\%Ti-1\%B às ligas do sistema Al-Si. Tecnologia em Metalurgia, Materiais e Mineração, v. 9, p.71-79, 2012.

ASSOCIAÇÃO BRASILEIRA DE NORMAS TÉCNICAS. NBR 11568: Determinação do tamanho de grão em materiais metálicos. Rio de Janeiro, 1990.

BIROL, Y.; Impact of grain size on mechanical properties of AISi7Mg0.3 alloy. Materials Science \& Engineering, v. 559, p. 394-400, 2012.

BIROL, Y.; Grain refinement and modification of Al-Si foundry alloys with B and Sr additions, Materials Science and Technology, v. 30, p. 1154-1161, 2014.

BOLZONI, L.; BABU, N.H.; Refinement of the grain size of the LM25 alloy (A356) by 96Al-2Nb-2B master alloy. Journal of Materials Processing Technology, v. 222, p. 219-223, 2015.

CHEN, Z.; WANG, T.; GAO, L.; FU, H.; LI, T.; Grain refinement and tensile properties improvements of alluminum foundry alloys by inoculation with Al-B master alloys. Material Science and Engineering $A$. v. 553, p. 32-36, 2012.

EASTON, M.; StJOHN, D.; Grain refinement of aluminum alloys: Part I. The nucleant and solute paradigms - A review of the literature. Metallurgical and Materials Transactions A, v. 30, p. 1613-1623, 1999.

GREER, A. L.; BUNN, A. M.; TRONCHE, A.; EVANS, P. V.; BRISTOW, D. J.; Modelling of inoculation of metallic melts: application to grain refinement of aluminium by Al-Ti-B. Acta Materialia, v. 48, p. 2823-2835, 2000.

QIU, D.; TAYLOR, J. A.; ZHANG, M. X.; KELLY, P. M.; A mechanism for the poisoning effect of silicon on the grain refinement of Al-Si alloys. Acta Materialia, v. 55, p 1447-1456 2007.

QUESTED, T. E.; Understanding mechanisms of grain refinement of aluminium alloys by inoculation. Materials Science and Technology, v. 20, p. 1357-1369, 2004.

QUESTED, T. E.; GREER, A. L.; The effect of the size distribution of inoculant particles on as-cast grain size in aluminium alloys. Acta Materialia, v. 52, p. 3859-3868, 2004.

MOHANTY, P. S.; GRUZLESKI, J. E.; Mechanism of grain refinement in aluminium. Acta Materialia. v. 43, p. 2001-2012, 1994.

NOWAK, M.; BOLZONI, L.; BABU, N.H.; Grain refinement of Al-Si alloys by Nb-B inoculantion. Part I: Concept development and effect on binary alloys. Materials and Design, v. 66, p. 366-375, 2015.

NOWAK, M.; YEOH, W.K.; BOLZONI, L.; BABU, N.H.; Development of Al-Nb-B master alloys using Nb and $\mathrm{KBF}_{4}$ powders. Materials and Design, v. 75, p. 40-45, 2015.

ZHANG, M. X., KELLY, P. M.; EASTON, M. A.; TAYLOR, J. A.; Crystallographic study of grain refinement in aluminum alloys using the edge-to-edge matching model. Acta Materialia, v. 53, p. 1427-1438, 2005. 